\title{
Marburg virus disease outbreak amidst COVID-19 in the Republic of Guinea: A point of contention for the fragile health system?
}

\author{
Osaretin Christabel Okonji ${ }^{a}$, Emeka Francis Okonji ${ }^{\mathrm{b}}$, Parvathy Mohanan ${ }^{\mathrm{c}}$, \\ Maryam Salma Babar ${ }^{\mathrm{d}}$, Amna Saleem ${ }^{\mathrm{e}}$, Uzzam Ahmed Khawaja ${ }^{\mathrm{e}}$, Mohammad Yasir Essar ${ }^{\mathrm{f}}$, \\ Mohammad Mehedi Hasan ${ }^{\text {g, }}$ \\ ${ }^{\text {a }}$ School of Pharmacy, University of the Western Cape, Bellville, South Africa \\ ${ }^{\mathrm{b}}$ School of Public Health, University of the Western Cape, Bellville, South Africa \\ ${ }^{\mathrm{c}}$ Department of General Medicine, Medical University of Sofia, Bulgaria \\ ${ }^{\mathrm{d}}$ Dubai Medical College, Dubai, United Arab Emirates \\ e Jinnah Medical and Dental College, Karachi, Pakistan \\ ${ }^{\mathrm{f}}$ Kabul University of Medical Sciences, Kabul, Afghanistan \\ ${ }^{g}$ Department of Biochemistry and Molecular Biology, Faculty of Life Science, Mawlana Bhashani Science and Technology University, Tangail, Bangladesh
}

\section{A R T I C L E I N F O}

\section{Keywords}

Marburg virus disease

COVID-19

Guinea

West Africa

Public health

\begin{abstract}
A B S T R A C T
In August 2021, the Marburg virus disease (MVD) outbreak was confirmed amid the coronavirus disease 2019 (COVID-19) pandemic in the Republic of Guinea. This is the first time it is detected in Guinea and West Africa. Marburg virus is one of the world's most threatening diseases, causing severe haemorrhagic fever, with a case fatality rate of $90 \%$. Currently, there are no vaccines and specific antiviral drugs for MVD. Technical teams and community health care workers that were set up as part of the recent Ebola virus disease (EVD) outbreak that was declared over on June 19, 2021, are now redeployed to support governments response activities of the MVD outbreak in the country. The MVD is an added burden to the fragile healthcare systems that are already overburdened with multiple reoccurring epidemics and the COVID-19 pandemic. Previous epidermic strategies are needed to contain the spread of the disease, amid the COVID-19 pandemic, so the health care systems are not overwhelmed. This commentary discusses the available evidence regarding the epidemic of MVD in Guinea amid the COVID-19 pandemic, and highlights the efforts, challenges to be prioritized, and provides evidence-based recommendations.
\end{abstract}

\section{Introduction}

Marburg virus disease (MVD) is a highly infectious and fatal disease caused by the Marburg virus (MV) and it is correlated with high case fatality rates (CFR 24-90\%). ${ }^{1}$ The Ministry of Health of the Republic of Guinea reported its first affirmed case of MVD on the 6 of August 2, 021 . $^{1}$ This is the first time MVD is detected in Guinea and in West Africa. ${ }^{1}$ The cause and reason for the outbreak are not known. The viral disease is a serious public health issue that has the potential to spread far and wide. The country recently declared an end to the Ebola virus disease (EVD) outbreak on June 19, 2021 that resurfaced in February $2021 .{ }^{1}$

The coronavirus 2019 (COVID-19) disease caused by SARS-CoV-2 has caused increased morbidity and mortality in several countries worldwide. Guinea reports its first case of COVID-19 in March 13, 2020. As of the October 23, 2021, more than 30,626 cases and 385 related deaths have been reported in the country. ${ }^{2}$ Although African countries have had a lower infection and mortality rate of COVID-19 as compared to other continents. Several factors including lack of access to diagnostic and incomplete reporting of deaths have been known to influence the low infection and mortality rate in Africa. ${ }^{3}$ However, Guinea has a

Abbreviations: MVD, Marburg virus disease; CFR, Case fatality rate; COVID-19, Coronavirus disease 2019; MV, Marburg virus; EVD, Ebola virus disease; SARSCoV-2, Severe acute respiratory syndrome coronavirus 2; WHO, World Health Organization; UNICEF, United Nations International Children's Emergency Fund; ALIMA, Alliance for Internal Medical Action.

* Corresponding author. Department of Biochemistry and Molecular Biology, Faculty of Life Science, Mawlana Bhashani Science and Technology University, Tangail, 1902, Bangladesh.

E-mail address: mehedi.bmb.mbstu@gmail.com (M.M. Hasan). 
fragile health care system which is further aggravated by numerous outbreaks, reoccurring epidemics and COVID-19 pandemic. ${ }^{1}$ The health crisis due to COVID-19 widespread will have a phenomenal effect on the worldwide economy hitting underdeveloped countries, such as Guinea.

Ever since MVD was detected, there have been two large outbreaks that happened concurrently and the outbreak was related to research laboratory work of African green monkeys imported from Uganda. ${ }^{4}$ In 2008, two unrelated sporadic cases occurred in travellers from the United States of America and the Netherland, during a visit to a cave inhabited by a large colony of Rousettus bat in Uganda. ${ }^{4}$ Consequent outbreaks and sporadic cases have been reported in Uganda, the Democratic Republic of Congo, Angola, Kenya and South Africa. ${ }^{4}$ The largest outbreak of MVD recorded so far occurred in Angola in 2005, and 374 cases were detected with 329 deaths with an $88 \%$ CFR. ${ }^{4}$ In Uganda, there has been four previous outbreaks in 2007, 2012, 2014 and 2017, the case fatality rate was between 27 and $100 \%$. Guinea reports the first outbreak in West Africa with only one case detected so far and the patient died. ${ }^{1}$ A total of 172 contacts have been identified and are been followed up. ${ }^{1}$ The region, Gueckedou where the MVD is confirmed happens to be the same region where cases of the EVD outbreak in June 2021 occurred and also the 2014-2016 West Africa EVD outbreak was originally detected. ${ }^{1}$

\section{Discussion}

Similar to coronavirus, marburg virus (MV) is generally transmitted from animal to human but it can spread between humans through direct contact with secretions, bodily fluids, blood and contaminated substances. ${ }^{1} \mathrm{MV}$ is disseminated in an individual through fruit bats and the Rousettus bat colonies reside in caves, therefore, humans contract this infection secondary to sustained exposure to mines or caves. In addition, healthcare workers acquire the illness due to needle stick injury and close association with patients who have negligent behaviour towards the safety protocols. ${ }^{1}$ Moreover, propinquity with the body of dead people in burial ceremonies also plays a role in the spread of MV. ${ }^{1}$

Since MVD, at an early stage has similar clinical presentations with COVID-19, this might worsen the problem. This can have a negative consequence, because there may be an overlap of symptoms between the two viral diseases, causing diagnosis and the treatment of a specific disease entity more complicated. For example, symptoms of COVID-19 which include lower respiratory distress, fever and neurological damage, this symptoms may overlap with MVD symptoms. ${ }^{5,6}$ This can worsen the health condition of the patient and therefore, lead to adverse and unfavourable treatment outcomes and worse prognosis particularly when the patient is diagnosed with SARS-CoV-2 infection, at the same time infected with MVD. Since there are no vaccines and specific antiviral drugs for MVD, treatment of the viral disease includes supportive care and management of co-infection. ${ }^{1}$

The COVID-19 pandemic caused several disruptions in many countries, which has resulted in surging cases of several infectious diseases. ${ }^{7-14}$ For instance in Guinea, the pandemic increased the difficulty in accessing health services and has also negatively affected surveillance, testing, treatment for other infectious diseases. ${ }^{15,16}$ The health systems were overburdened - with increased hospital workload thus influencing patient care. This could deter or delay the treatment and management of patients with MVD and quick linkage to MVD care could be delayed during the pandemic since hospitals are focusing mostly on COVID-19 patients. In addition, the allocations of health resources for MVD care could be reduced, and circumstances surrounding the MVD care could exacerbate since Guinea has a shortage of health care workers and infrastructure. ${ }^{17}$ Therefore, the national health care systems in the country can be easily overwhelmed by the concurrent rise in both COVID-19 and MVD cases. In addition, the total health funding expenditures percent of GDP for Guinea is $3.93 \% .{ }^{18}$ On a per capital basis, Guinea spends $\$ 38.32$ on health, ${ }^{18}$ which is well below the recommended US $\$ 86$ per capita low- and middle-income country bench mark required to provide key health services. ${ }^{19}$ The health funding for Guinea is quite low and can influence the management of both COVID-19 and MVD cases in the country.

\section{Challenges}

Currently, there are challenges which include a missing high-risk contact of the MVD case and the inadequate infection prevention and control measures in health facilities in the country which indicates that there are gaps in response capacity that require urgent attention. ${ }^{20} \mathrm{Be}$ sides, the source of infection of the index case remains unknown. The Nzérékoré region borders Sierra Leone, and Liberia, hence frequent cross-border movements of people could result in further disease spread to neighbouring countries. In addition, the country is already overburdened with multiple outbreaks, for example, COVID-19, yellow fever, measles and vaccine-derived polio. In addition, lack of access to basic primary health care services, transportation and inadequate ambulance services have always been a key challenge in countries in the West African region. West African countries including Guinea lack the public health infrastructure needed to manage infectious disease outbreaks on a structural level. ${ }^{21}$ Disease surveillance is not strong in the affected countries, and many health centers are already plagued by logistical challenges. ${ }^{15,21,22}$

\section{Efforts}

In response to the epidemic, the Ministry of Health in Guinea in collaboration with several organizations including the World Health Organization (WHO), United Nations International Children's Emergency Fund (UNICEF), Alliance for Internal Medical Action, (ALIMA), United States Centers for Disease Control and Prevention, Red cross, the International Organization for Migration and other partners, are working together to prevent additional new cases of MVD through response activities such as contact tracing and active case searching in health facilities and affected communities, enhanced surveillance, risk communication, community engagement and other infection prevention and control activities since there are no vaccines and treatment for MVD. ${ }^{1}$ Besides, the WHO technical team that remains in the country to assist the government's implementation of a post -EVD plan together with the community health workers that was set up as part of the recent EVD outbreak that was declared over in June 19, 2021, are now redeployed to support governments response activities of the MVD outbreak in the country. ${ }^{1}$ The Ebola control system that has been placed in the country is an added advantage and turning out important to the swift emergency response to the MVD outbreak. ${ }^{6,20}$ task force is also working closely with countries sharing borders with the Gueckedou to monitor any cross-border spread of the virus.

\section{Recommendations and conclusion}

Even though the country has been able to curtain several outbreaks such as Ebola and Lassa fever, the fragile health systems that are exacerbated by multiple disease outbreaks recurring viral haemorrhagic epidemics and the COVID-19 pandemic needs to be strengthened. Government should increase health funding as well as increase the number of beds and health care workforce. It is crucial to promote epidemiology, community engagement, and integrated surveillance programs, especially in the affected region in the country. Healthcare professionals, policymakers and researchers should be aware of the coexistence of both MVD and COVID-19. Healthcare workers is important for the continuation of care in the country. With more infectious variants such as the Delta variant of SARS-CoV-2 emerging, along with the MVD, it is crucial to ensure that medical professionals are wellequipped with protective equipment. Appropriate laboratory diagnosis is necessary to differentiate MVD and COVID-19 cases. Community leaders and health workers in affected areas should create awareness 
about the risk factors of MVD, protective measures about thorough cooking of animal products such as meat and blood before consumption, avoidance of contact with fruit bats, monkeys and apes, wearing of mask and gloves if need to come in contact with these animals why observing regular hand hygiene and prompt and safe burial practices for people who have died from MVD. The country-specific guidelines provided by the WHO should be used to prevent MVD in endemic areas in the country. The government and stakeholders should work together and ensure the MVD epidemic during the COVID-19 pandemic, is quickly contained so that the health care systems are not overwhelmed. Even though the scale of the COVID-19 pandemic exceeds most infectious diseases in Africa, the World Health Organization should strengthen coordination with regards MVD in endemic countries and should ensure continued financial support in order to minimise the re-emergence of this fatal disease.

\section{Author contributions}

OCO came up with the idea and conceptualization of the study. OCO, EFO, PM, MSB, AS, UAK, MMH and MYE were responsible for drafts of the manuscript. OCO, MMH and MYE made the critical review of the manuscript. All authors read and approved the final manuscript. All authors revised and approved the final draft.

\section{Financial support}

No financial support was acquired for this article.

\section{Declaration of competing interest}

The authors declare no conflict of interest.

\section{Acknowledgments}

Not applicable.

\section{References}

1 World Health Organisation (WHO). Marburg Virus Disease - Guinea; 2021. https ://www.who.int/emergencies/disease-outbreak-news/item/2021-Don331. Accessed September 17, 2021

2 World Health Organisation (WHO). Guinea: WHO coronavirus disease (COVID-19) dashboard with vaccination data. https://covid19.who.int/region/afro/country/gn; 2021. Accessed September 17, 2021.

3 Okonji EF, Okonji OC, Mukumbang FC, Wyk B Van. Understanding varying COVID19 mortality rates reported in Africa compared to Europe, Americas and Asia. Trop Med Int Health. 2021;26(7):716-719. https://doi.org/10.1111/TMI.13575.
4 World Health Organisation (WHO). Marburg Virus Disease; 2021. https://www.who. int/news-room/fact-sheets/detail/marburg-virus-disease. Accessed September 17, 2021.

5 Çavdaroğlu S, Hasan MM, Mohan A, et al. The spread of Yellow fever amidst the COVID-19 pandemic in Africa and the ongoing efforts to mitigate it. J Med Virol. 2021;93(9):5223-5225. https://doi.org/10.1002/JMV.27027.

6 Khan FMA, Hasan MM, Kazmi Z, et al. Ebola and COVID-19 in Democratic Republic of Congo: grappling with two plagues at once. Trop Med Health. 2021;49(1):1-4. https://doi.org/10.1186/S41182-021-00356-6, 2021491.

7 Mohan A, Temitope RA, Çavdaroğlu S, et al. Measles returns to the Democratic Republic of Congo: a new predicament amid the COVID-19 crisis. J Med Virol. 2021; 93(10):5691-5693. https://doi.org/10.1002/JMV.27137.

8 Hasan MM, Costa AC, dos S, Xenophontos E, et al. Lassa fever and COVID-19 in Africa: a double crisis on the fragile health system. J Med Virol. 2021;93(10): 5707-5709. https://doi.org/10.1002/JMV.27169.

9 Hasan MM, Mohanan P, Emmanuella N, et al. Challenges of HIV amidst COVID-19 in Africa: can we conquer them? J Med Virol. 2021. https://doi.org/10.1002/ JMV. 27276.

10 Khan FMA, Kazmi Z, Hasan MM, et al. Resurgence of tuberculosis amid COVID-19 in Peru: associated risk factors and recommendations. Int J Health Plann Manag. 2021. https://doi.org/10.1002/HPM.3291.

11 Ghosh S, Patelia S, Hasan MM, Ghosh A, Jain S, Patel T. Drug Resistant White Fungus: Another Catastrophic Fungus Emergence amidst COVID-19 in India. 2021. https://doi. org/10.1080/20477724.2021.1960762.

12 Yousaf A, Khan FMA, Hasan MM, Ullah I, Bardhan M. Dengue, measles, and COVID19: a threefold challenge to public health security in Pakistan. Ethics, Med Public Heal. 2021;19:100704. https://doi.org/10.1016/J.JEMEP.2021.100704.

13 Abbas KS, Kaml MBS, Metry MAF, et al. Hepatitis C liver disease during COVID-19 pandemic in Egypt: challenges and way forward. Ethics, Med Public Heal. 2021;19: 100702. https://doi.org/10.1016/J.JEMEP.2021.100702.

14 Ghazi BK, Rackimuthu S, Wara UU, et al. Rampant increase in cases of mucormycosis in India and Pakistan: a serious cause for concern during the ongoing COVID-19 pandemic. Am J Trop Med Hyg. 2021;1(aop). https://doi.org/10.4269/AJTMH.210608.

15 Aborode AT, Hasan MM, Jain S, et al. Impact of poor disease surveillance system on COVID-19 response in africa: time to rethink and rebuilt. Clin Epidemiol Glob Heal. 2021;12:100841. https://doi.org/10.1016/J.CEGH.2021.100841.

16 Saalim K, Sakyi KS, Fatema-Tuz-Zohra, et al. Reported health and social consequences of the COVID-19 pandemic on vulnerable populations and implemented solutions in six West African countries: a media content analysis. PLoS One. 2021;16(6), e0252890. https://doi.org/10.1371/JOURNAL.PONE.0252890.

17 World Health Organization (WHO). Global Health Workforce Statistics Database-; 2021. https://www.who.int/data/gho/data/themes/topics/indicator-group/indica tor-group-details/GHO-medical-doctors. Accessed September 17, 2021.

18 Data- World Bank. Current health expenditure (\% of GDP). https://data.worldbank. org/indicatore/SH.XPD.CHEX.GD.ZS; September 2021. Accessed September 17, 2021.

19 Mcintryre D, Meheus F, Rottingen J. What level of domestic government health expenditure should we aspire to for universal health coverage? Health Econ Pol Law. 2017;12(2):125-137. https://doi.org/10.1017/S1744133116000414.

20 Weekly Bulletins on Outbreaks and Other Emergencies | WHO | Regional Office for Africa. https://www.afro.who.int/health-topics/disease-outbreaks/outbreaks-andother-emergencies-updates. Accessed September 17, 2021.

21 Boozary AS, Farmer PE, Jha AK. The Ebola outbreak, fragile health systems, and quality as a cure. J Am Med Assoc. 2014;312(18):1859-1860. https://doi.org/ 10.1001/JAMA.2014.14387.

22 Buesen AG, Stevens PE, Bromberg M, Kelber ST. The Ebola epidemic in West Africa: challenges, opportunities, and policy priority areas. Nurs Outlook. 2015;63(1):30-40. https://doi.org/10.1016/J.OUTLOOK.2014.12.013. 